\title{
Factors associated with serum total homocysteine level in type 2 diabetes
}

\author{
Yumi Masuda $\cdot$ Akira Kubo $\cdot$ Akatsuki Kokaze \\ Masao Yoshida $\cdot$ Nobuki Fukuhara • \\ Yutaka Takashima
}

Received: 21 June 2007/ Accepted: 18 December 2007/Published online: 29 March 2008

(C) The Japanese Society for Hygiene 2008

\begin{abstract}
Objectives The aim of this study was to investigate the determinants of serum total homocysteine level (tHcy) in patients with type 2 diabetes mellitus (DM) according to sex. Methods A total of 1,276 Japanese, diabetics $(n=280)$ with a control group of non-diabetics $(n=996)$, were enrolled into the study from 2003 to 2005 . This crosssectional study was conducted for all the subjects, using personal data regarding clinical characteristics and lifestyle. Multiple regression analysis was performed to analyze the association of tHcy with selected factors.

Results In diabetic subjects, estimated glomerular filtration rate (eGFR) and serum creatinine levels (Cre), even those within the normal range, were strongly associated with tHcy after adjustment in both sexes; the standardized partial regression coefficient of eGFR for tHcy was $-0.251, \quad(p=0.001)$ in diabetic men and -0.523 , $(p<0.001)$ in diabetic women. Furthermore, the eGFR of the diabetics, except patients with nephropathy, also had significant association with tHcy in both sexes. Fasting plasma glucose levels and serum triglyceride levels were
\end{abstract}

Y. Masuda $(\bowtie) \cdot$ M. Yoshida · Y. Takashima

Department of Public Health,

Kyorin University School of Medicine, 6-20-2 Shinkawa,

Mitaka, Tokyo 181-8611, Japan

e-mail: lyumi@mvd.biglobe.ne.jp

A. Kubo · N. Fukuhara

Takanawa Medical Clinic, 2-16-29 Takanawa,

Minato, Tokyo 108-0074, Japan

e-mail: tmc@mx1.alpha-web.ne.jp

\section{A. Kokaze}

Department of Public Health,

Showa University School of Medicine,

1-5-8 Hatanodai, Shinagawa, Tokyo 142-8555, Japan strongly associated with tHcy in diabetic men only. HbA1c was also associated with tHcy in diabetic men only, though not as significantly. Age and presence of hypertension were significantly associated with tHcy in women.

Conclusions This study suggests that there are some differences in the factors associated with tHcy between diabetics and non-diabetics, and between the sexes. There is, therefore, circumstantial evidence that elevated tHcy should be evaluated clinically. Because tHcy was strongly associated with eGFR and Cre, even within the normal ranges, tHcy may have important implications regarding the microangiopathy of the kidney and atherosclerosis.

Keywords Homocysteine - Type 2 diabetes mellitus . Lifestyle $\cdot$ Estimated glomerular filtration rate .

Serum creatinine

\section{Introduction}

Homocysteine is a sulfur-containing amino acid formed during metabolism of the essential amino acid methionine $[1,2]$. Serum total homocysteine concentration (tHcy) has been recognized as an atherogenic factor, promoting oxidative stress, inflammation, thrombosis, endothelial dysfunction, and cell proliferation [2-4]. In addition, many previous epidemiologic studies suggest that elevated tHcy is a new, strong, and independent risk factor of coronary heart disease (CHD) and stroke [5-7]. Also in Japan, several previous studies reported that tHcy was an independent risk factor of ischemic stroke $[8,9]$.

It has also been reported that several factors, including age, gender, smoking, alcohol consumption, malignancies, thyroid disease, renal failure, nutrition (deficiency of folic acid, Vitamin B6 and B12), medication, and gene type of 
methylene tetrahydrofolate reductase (MTHFR) can affect tHcy [10-12]; Jacques [13-15]. Thus, the changes in tHcy might derive from a combination of these factors [10]. As regards the effects of age and gender, it was clearly shown that tHcy increased with age, and was higher in males than in females [16].

Diabetic patients have increased risk of vascular complications or CHD, since hyperglycemia leads to vascular dysfunction [17, 18]. In Japan, diabetics have a two to fourfold greater risk of vascular disease occurrence as compared with non-diabetics [19]. In recent years, it has been suggested that atherosclerosis develops markedly even in the phase of mild impairment of glucose tolerance [20]. In addition, several studies have shown the positive and negative association between tHcy and pathophysiology of diabetes mellitus (DM) [14, 21-24]. In Japan, some studies have reported the factors associated with tHcy in DM [25]. However, the clinical guideline for lowering tHcy has not yet been fully established in Japanese community healthcare activities. Accordingly, it will be necessary that the determinants of tHcy in DM patients will be concurrently analyzed by multi-factors.

The aim of this study was, first, to investigate the crosssectional association of tHcy with DM in middle-aged and old-aged Japanese according to sex. The second objective was to clarify the associations of tHcy with other various biomarkers or health indices. Moreover, the third objective was to clarify which type of DM treatment and complication could further specifically affect tHcy in DM patients.

\section{Subjects and methods}

\section{Subjects}

A total of 1,276 individuals, who had visited a medical clinic in Tokyo, from 1st August 2003 to 31st July 2005, were enrolled in the study. They consisted of 587 males and 689 females, who had had a complete medical checkup or diabetic outpatient care at the clinic. Two hundred and eighty of the 1,276 subjects were diagnosed with type 2 DM. No one suffered from type 1 or other DM types. The diabetic patients in this study were treated in one of three ways: with special dietetic therapy and exercise by dieticians and exercise therapists; with oral agents; or with insulin therapy. All patients received the complete course of their therapy. Patients with malignancy or thyroid disease were excluded from the study. One-hundred and eighty-six of the 1,276 subjects had experienced myocardial infarction (MI) or cerebral infarction (CI). All the subjects gave written informed consent before participating in the study. This study was approved by the Human Ethics Committee of Kyorin University School of Medicine.
Clinical measurements and questionnaire

All subjects underwent the following physical examinations: tHcy, fasting plasma glucose level (FPG), hemoglobin A1c level (HbA1c), total cholesterol level in serum (TC), serum triglyceride level (TG), serum creatinine level (Cre), blood pressure (BP), and body mass index (BMI). Furthermore, estimated glomerular filtration rate (eGFR) was calculated by the modification of diet in renal disease (MDRD) method [26].

Serum total homocysteine concentration was determined by HPLC (LC-9A, Shimadzu, Kyoto, Japan) [11]. Cre was assayed by an enzyme method. The glucose-hydrogenase method was used for plasma glucose assay [27]. The latex agglutination turbidimetric immunoassay method was used for $\mathrm{HbA1c}$ assay [28]. TC was determined by the cholesterol dehydrogenase (UV-End) method [29]. TG was determined by an enzyme method [30] and transformed to a logarithm because of non-normal distribution. Brachial blood pressure of the subjects was measured by use of a mercury sphygmomanometer in the sitting position after sufficient rest. BP was classified into the following three categories: hypertension, systolic blood pressure $(\mathrm{SBP}) \geq 140 \mathrm{mmHg}$, or diastolic blood pressure (DBP) $\geq 90 \mathrm{mmHg}$; high level of normal range, $130 \mathrm{mmHg} \leq \mathrm{SBP}<140 \mathrm{mmHg}$, and/or $80 \mathrm{mmHg} \leq \mathrm{DBP}<90 \mathrm{mmHg}$ except for hypertension; and normal, SBP $<130 \mathrm{mmHg}$ and $\mathrm{DBP}<80 \mathrm{mmHg}$ [31].

Personal information regarding the subjects was collected from their medical records and from a questionnaire.

Identification of DM cases was fulfilled according to diagnostic criteria set by Japan Diabetes Society recommendations [32]. Presence of MI or CI, contents of treatment regimen for DM and complication status of DM characterized by neuropathy, retinopathy, or nephropathy, were also confirmed from subjects' medical records at the point of the examinations. In this study, patients with nephropathy had $300 \mathrm{mg} /$ day or more urinary protein.

An interview-based questionnaire was conducted to investigate each subject's background and lifestyle. The questionnaire included questions concerning the issues:

1 smoking habits-non-smokers or current smokers; and

2 drinking habits-non/occasional drinkers or habitual drinkers.

Statistical analysis

In this study, the data of age, gender, tHcy, FPG, HbA1c, C, logarithmic TG (logTG), Cre, eGFR, BMI, BP (normal $=0$, high level of normal range $=1$, hypertension $=2$ ), current smoking habit (non-smokers $=0$, current smokers $=1$ ), routine drinking habit (non-or occasional drinkers $=0$, habitual drinkers $=1$ ), $\mathrm{DM}$ 
(non-diabetics $=0$, diabetics $=1$ ), diabetic complication (neuropathy, retinopathy, nephropathy), types of treatment regimen for $\mathrm{DM}$ (only diet and exercise $=0$, oral agent $=1$, insulin therapy $=2$ ), previous history of $\mathrm{MI}$ or CI (not yet $=0$, presence of $\mathrm{MI}$ or $\mathrm{CI}=1$ ), and information about taking antihypertensive agent (no $=0$, routine intake $=1$ ) or lipid-lowering agent (no $=0$, routine intake $=1$ ) were subjected to analyses as a crosssectional study. In addition to the analyses for all the subjects $(n=1,276)$, the DM group $(n=280)$ and the non-DM group $(n=996)$ were also conducted as the subgroups. Multiple regression analysis was performed to analyze the association of tHcy with selected factors. The 0.05 level was regarded as the cut-off point of statistical significance.

All the statistical analyses were performed using SAS statistical software for Windows, version 8.2.

\section{Results}

Detailed characteristics of subjects are shown in Table 1. The mean levels of tHcy were $11.26 \mu \mathrm{mol} / \mathrm{l}$ for males and $8.60 \mu \mathrm{mol} / 1$ for females. tHcy was higher in males than in females, and was higher in DM than in non-DM.

As shown in Table 2, in both sexes, DM was significantly associated with increased tHcy after adjustment for age and Cre; partial regression coefficient $(\beta) \pm$ standard error in tHcy between DM and non-DM was $1.409 \pm 0.458(p=0.002)$ in males and $1.004 \pm 0.275$ $(p<0.001)$ in females. The association of HbA1c with tHcy was significant in males only after adjustment for multi-variables. Substituted DM or FPG as variables for HbA1c in Model B produced the same results: standard partial regression coefficient of DM for tHcy was 0.107 $(p<0.05)$ in males and $0.060(p>0.05)$ in females; that

Table 1 Basic characteristic data for all subjects

\begin{tabular}{|c|c|c|c|c|c|c|}
\hline \multirow[b]{2}{*}{$N$} & \multicolumn{3}{|l|}{ Males } & \multicolumn{3}{|l|}{ Females } \\
\hline & $\begin{array}{l}\text { DM } \\
196\end{array}$ & $\begin{array}{l}\text { Non-DM } \\
391\end{array}$ & $p$ value & $\begin{array}{l}\text { DM } \\
84\end{array}$ & $\begin{array}{l}\text { Non-DM } \\
605\end{array}$ & $p$ value \\
\hline Homocysteine $(\mu \mathrm{mol} / \mathrm{l})^{\mathrm{a}}$ & $12.28 \pm 7.23$ & $10.75 \pm 3.39$ & $0.005^{\mathrm{b}}$ & $9.99 \pm 4.39$ & $8.41 \pm 2.40$ & $0.002^{\mathrm{b}}$ \\
\hline $\mathrm{Age}^{\mathrm{a}}$ & $60.76 \pm 10.37$ & $56.14 \pm 13.12$ & $<0.001^{\mathrm{b}}$ & $64.73 \pm 10.57$ & $56.38 \pm 13.40$ & $<0.001^{\mathrm{t}}$ \\
\hline Fasting plasma glucose $(\mathrm{mg} / \mathrm{dl})^{\mathrm{a}}$ & $173.58 \pm 82.29$ & $90.13 \pm 8.91$ & $<0.001^{\mathrm{b}}$ & $172.88 \pm 85.63$ & $87.10 \pm 8.62$ & $<0.001^{\mathrm{t}}$ \\
\hline $\operatorname{HbA} 1 \mathrm{c}(\%)^{\mathrm{a}}$ & $7.72 \pm 1.50$ & $5.10 \pm 0.35$ & $<0.001^{\mathrm{b}}$ & $7.90 \pm 1.86$ & $5.13 \pm 0.36$ & $<0.001^{\mathrm{t}}$ \\
\hline Total cholesterol $(\mathrm{mg} / \mathrm{dl})^{\mathrm{a}}$ & $211.29 \pm 35.78$ & $210.13 \pm 42.70$ & $0.730^{\mathrm{b}}$ & $230.38 \pm 35.99$ & $219.79 \pm 42.46$ & $0.030^{\mathrm{r}}$ \\
\hline Triglyceride $(\mathrm{mg} / \mathrm{dl})^{\mathrm{a}}$ & $156.12 \pm 105.13$ & $135.18 \pm 98.40$ & $0.018^{\mathrm{b}}$ & $160.04 \pm 111.59$ & $91.99 \pm 59.20$ & $<0.001^{\mathrm{t}}$ \\
\hline Serum creatinine $(\mathrm{mg} / \mathrm{dl})^{\mathrm{a}}$ & $0.88 \pm 0.27$ & $0.86 \pm 0.15$ & $0.423^{\mathrm{b}}$ & $0.71 \pm 0.68$ & $0.65 \pm 0.12$ & $0.468^{\mathrm{b}}$ \\
\hline eGFR $\left(\mathrm{ml} / \mathrm{min} / 1.73 \mathrm{~m}^{2}\right)^{\mathrm{a}}$ & $70.38 \pm 17.10$ & $70.47 \pm 14.34$ & $0.949^{\mathrm{b}}$ & $73.73 \pm 22.11$ & $71.95 \pm 14.55$ & $0.483^{\mathrm{b}}$ \\
\hline Body mass index $\left(\mathrm{kg} / \mathrm{m}^{2}\right)^{\mathrm{a}}$ & $23.81 \pm 4.00$ & $23.51 \pm 2.92$ & $0.376^{\mathrm{b}}$ & $23.42 \pm 4.79$ & $21.72 \pm 3.31$ & $0.005^{\mathrm{b}}$ \\
\hline \multicolumn{7}{|l|}{ Blood pressure $(\%)$} \\
\hline $\mathrm{N}$ & $20.41(n=40)$ & $46.83(n=183)$ & $<0.001^{\mathrm{c}}$ & $13.10(n=11)$ & $62.22(n=376)$ & $<0.001^{\mathrm{C}}$ \\
\hline B & $18.88(n=37)$ & $11.64(n=46)$ & & $17.86(n=15)$ & $9.74(n=59)$ & \\
\hline $\mathrm{H}$ & $60.71(n=119)$ & $41.53(n=162)$ & & $69.05(n=58)$ & $28.03(n=170)$ & \\
\hline \multicolumn{7}{|l|}{ Current smoking habits $(\%)$} \\
\hline$(-)$ & $68.37(n=134)$ & $81.03(n=317)$ & $<0.001^{\mathrm{c}}$ & $86.90(n=73)$ & $90.89(n=550)$ & $0.245^{\circ}$ \\
\hline$(+)$ & $31.63(n=62)$ & $18.97(n=74)$ & & $13.10(n=11)$ & $9.11(n=55)$ & \\
\hline \multicolumn{7}{|l|}{ Routine drinking habits $(\%)$} \\
\hline$(-)$ & $35.20(n=69)$ & $26.92(n=105)$ & $0.039^{\mathrm{c}}$ & $75.00(n=63)$ & $57.95(n=351)$ & $0.003^{\circ}$ \\
\hline$(+)$ & $64.80(n=127)$ & $73.08(n=286)$ & & $25.00(n=21)$ & $42.05(n=254)$ & \\
\hline No history of vascular event (\%) & $80.10(n=157)$ & $89.26(n=349)$ & $0.021^{\mathrm{c}}$ & $76.19(n=64)$ & $92.56(n=560)$ & $<0.001^{\mathrm{C}}$ \\
\hline History of only MI (\%) & $12.76(n=25)$ & $7.67(n=30)$ & & $16.67(n=14)$ & $5.29(n=32)$ & \\
\hline History of only CI (\%) & $4.08(n=8)$ & $1.79(n=7)$ & & $7.14(n=6)$ & $1.49(n=9)$ & \\
\hline History of MI and CI (\%) & $3.06(n=6)$ & $1.28(n=5)$ & & $0.00(n=0)$ & $0.66(n=4)$ & \\
\hline
\end{tabular}

eGFR, estimated glomerular filtration rate by MDRD method; $\mathrm{N}$, normal as $\mathrm{SBP}<130 \mathrm{mmHg}$, and $\mathrm{DBP}<80 \mathrm{mmHg}$; $\mathrm{B}$, high level of normal range as $130 \mathrm{mmHg} \leq \mathrm{SBP}<140 \mathrm{mmHg}$, and/or $80 \mathrm{mmHg} \leq \mathrm{DBP}<90 \mathrm{mmHg}$ except for hypertension; H, hypertension as $\mathrm{SBP} \geq 140$ $\mathrm{mmHg}$, or DBP $\geq 90 \mathrm{mmHg}$; MI, myocardial infarction; CI, cerebral infarction

${ }^{\text {a }}$ Mean and standard deviations are indicated

b $p$ value by Welch's $t$ test

${ }^{c} p$ value by Chi-square test 
Table 2 Correlations with serum total homocysteine concentration level in all subjects, calculated by multiple regression analysis

\begin{tabular}{|c|c|c|c|c|c|c|c|c|}
\hline & \multicolumn{4}{|l|}{ Male $(n=587)$} & \multicolumn{4}{|l|}{ Female $(n=689)$} \\
\hline & $\begin{array}{l}\text { Partial } \\
\text { regression } \\
\text { coefficient } \\
(\beta) \pm \mathrm{SE} \\
\text { Model } \mathrm{A}^{\mathrm{d}}\end{array}$ & 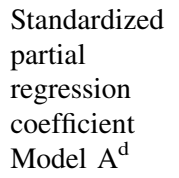 & $\begin{array}{l}p \text { value } \\
\text { of } \\
\text { model } \\
\mathrm{A}^{\mathrm{d}}\end{array}$ & $\begin{array}{l}\text { Standardized } \\
\text { partial } \\
\text { regression } \\
\text { coefficient } \\
\text { Model B }^{\mathrm{e}}\end{array}$ & $\begin{array}{l}\text { Partial } \\
\text { regression } \\
\text { coefficient } \\
(\beta) \pm \mathrm{SE} \\
\text { Model } \mathrm{A}^{\mathrm{d}}\end{array}$ & $\begin{array}{l}\text { Standardized } \\
\text { partial } \\
\text { regression } \\
\text { coefficient } \\
\text { Model } \mathrm{A}^{\mathrm{d}}\end{array}$ & $\begin{array}{l}p \text { value } \\
\text { of } \\
\text { model } \\
\mathrm{A}^{\mathrm{d}}\end{array}$ & $\begin{array}{l}\text { Standardized } \\
\text { partial } \\
\text { regression } \\
\text { coefficient } \\
\text { Model } \mathrm{B}^{\mathrm{e}}\end{array}$ \\
\hline Age & $0.012 \pm 0.018$ & 0.027 & 0.516 & -0.090 & $0.039 \pm 0.007$ & 0.174 & $<0.001$ & 0.058 \\
\hline DM & $1.409 \pm 0.458$ & 0.128 & 0.002 & - & $1.004 \pm 0.275$ & 0.120 & $<0.001$ & - \\
\hline FPG (mg/dl) & $0.018 \pm 0.003$ & 0.223 & $<0.001$ & - & $0.006 \pm 0.002$ & 0.085 & 0.010 & - \\
\hline HbA1c (\%) & $0.559 \pm 0.141$ & 0.167 & $<0.001$ & $0.115^{*}$ & $0.251 \pm 0.078$ & 0.110 & 0.001 & 0.071 \\
\hline $\mathrm{TC}(\mathrm{mg} / \mathrm{dl})$ & $0.021 \pm 0.005$ & 0.163 & $<0.001$ & 0.064 & $0.005 \pm 0.002$ & 0.071 & 0.033 & 0.064 \\
\hline $\log \mathrm{TG}(\mathrm{mg} / \mathrm{dl})$ & $0.837 \pm 0.370$ & 0.095 & 0.024 & -0.038 & $0.393 \pm 0.175$ & 0.076 & 0.025 & -0.037 \\
\hline Cre $(\mathrm{mg} / \mathrm{dl})$ & $6.015 \pm 1.118$ & 0.225 & $<0.001$ & - & $5.809 \pm 0.343$ & 0.552 & $<0.001$ & - \\
\hline eGFR & $-0.067 \pm 0.015$ & -0.195 & $<0.001$ & $-0.280 *$ & $-0.060 \pm 0.007$ & -0.331 & $<0.001$ & $-0.271 * *$ \\
\hline $\mathrm{BP}$ & $0.635 \pm 0.255$ & 0.111 & 0.013 & -0.018 & $0.458 \pm 0.111$ & 0.149 & $<0.001$ & $0.158^{*}$ \\
\hline a-h agent & $1.489 \pm 0.501$ & 0.211 & $<0.001$ & $0.181 *$ & $0.397 \pm 0.233$ & 0.059 & 0.092 & -0.026 \\
\hline BMI $\left(\mathrm{kg} / \mathrm{m}^{2}\right)$ & $0.081 \pm 0.045$ & 0.075 & 0.076 & 0.063 & $0.070 \pm 0.028$ & 0.096 & 0.013 & 0.060 \\
\hline Smoking $^{\mathrm{a}}$ & $1.433 \pm 0.519$ & 0.116 & 0.006 & 0.057 & $0.678 \pm 0.325$ & 0.068 & 0.038 & $0.084 *$ \\
\hline Drinking $^{\mathrm{b}}$ & $-0.376 \pm 0.473$ & -0.033 & 0.428 & $-0.115^{*}$ & $-0.285 \pm 0.191$ & -0.049 & 0.135 & -0.012 \\
\hline $\mathrm{V}$ event $^{\mathrm{c}}$ & $-0.305 \pm 0.634$ & -0.020 & 0.631 & -0.056 & $0.939 \pm 0.307$ & 0.101 & 0.002 & 0.080 \\
\hline
\end{tabular}

$\mathrm{DM}$, non-diabetics $=0$, diabetics $=1 ;$ Cre, serum creatinine; eGFR, estimated glomerular filtration rate by MDRD method $(\mathrm{ml} / \mathrm{min} / 1.73 \mathrm{~m})^{2}$; $\mathrm{BP}$, blood pressure $(\mathrm{N}=0$; normal as $\mathrm{SBP}<130 \mathrm{mmHg}$, and $\mathrm{DBP}<80 \mathrm{mmHg}, \mathrm{B}=1$, high level of normal range as $130 \mathrm{mmHg} \leq$ $\mathrm{SBP}<140 \mathrm{mmHg}$, and/or $80 \mathrm{mmHg} \leq \mathrm{DBP}<90 \mathrm{mmHg}$ except for hypertension, $\mathrm{H}=2$; Hypertension as $\mathrm{SBP} \geq 140 \mathrm{mmHg}$, or $\mathrm{DBP}$ $\geq 90 \mathrm{mmHg}$ ); a-h agent, anti-hypertensive agent $($ no $=0$, routine intake $=1)$

a Smoking, smoking habits (non-smokers $=0$, current smokers $=1$ )

${ }^{\mathrm{b}}$ Drinking, routine drinking habits (non-or occasional drinkers $=0$, habitual drinkers $=1$ )

${ }^{c} \mathrm{~V}$ event, history of myocardial infarction or cerebral infarction (not yet $=0$, have past history $=1$ )

${ }^{\mathrm{d}}$ Model A was adjusted for age and Cre, however, that of eGFR was adjusted for age

e Model B was adjusted for multi-variables (age, HbA1c, TC, logTG, eGFR, BP, a-h agent, BMI, smoking, drinking and V event)

$* p$ value $<0.05 ; * * p$ value $<0.001$

of FPG for tHcy was $0.098(p<0.05)$ in males and 0.047 $(p>0.05)$ in females, (data not shown in Table 2). eGFR had the strongest association with tHcy in both sexes.

As shown in Table 3, eGFR and Cre had a strong association with tHcy in both sexes according to multiple regression analysis, irrespective of presence or absence of DM. Furthermore, eGFR of the diabetics except patients with nephropathy had also strong association with tHcy in both sexes $(p=0.023$ and $p=0.017$ in males and females, respectively, data not shown in Table 3). eGFR and Cre had also strong association with tHcy after adjustment for Model B. Table 3 shows that the factors except eGFR and Cre associated with tHcy were different between diabetics and non-diabetics in males. The FPG and the $\log$ TG after adjustment for Model A were significantly positively associated with tHcy in diabetic males only. In contrast, taking anti-hypertension agents, BP, and drinking habits were associated with tHcy in non-diabetic males.
However, there was no difference in the factors associated with tHcy between DM and non-DM in females. Age, BP, Cre and eGFR were significantly associated with tHcy in females. With regard to association with lifestyle, current smoking habits had no significant association, but they had a close positive relation with tHcy in diabetic males, whereas drinking habits were negatively associated with tHcy in non-diabetic males.

In Table 4, tHcy is compared among different subgroups by type of DM treatment and diabetic complications according to analysis of covariance. As regards the association with type of treatment, tHcy was highest in subjects receiving insulin therapy and lowest in those using only diet and exercise, after adjustment for age, $\mathrm{HbAlc}$, and Cre. It was found that nephropathy was significantly associated with tHcy after adjustment for age and HbAlc.

Even after further adjustment for past history of cardiovascular events and intake of metformin, one of the 
Table 3 Correlations with serum total homocysteine concentration level in diabetics and non-diabetic subjects according to multiple regression analysis

\begin{tabular}{|c|c|c|c|c|c|c|c|c|c|}
\hline & \multicolumn{5}{|l|}{$\mathrm{DM}(n=196)$} & \multicolumn{4}{|l|}{ Non-DM $(n=391)$} \\
\hline & $\begin{array}{l}\text { Partial } \\
\text { regression } \\
\text { coefficient } \\
(\beta) \pm \mathrm{SE} \\
\left(\text { Model } \mathrm{A}^{\mathrm{d}}\right)\end{array}$ & $\begin{array}{l}\text { Stan } \\
\text { parti } \\
\text { regr } \\
\text { coef } \\
\text { (Mo }\end{array}$ & $\begin{array}{l}\text { ardized } \\
\text { sion } \\
\text { ient } \\
\left.1 \mathrm{~A}^{\mathrm{d}}\right)\end{array}$ & $\begin{array}{l}p \text { value } \\
\left(\text { Model } A^{\mathrm{d}}\right)\end{array}$ & $\begin{array}{l}\text { Standardized } \\
\text { partial regression } \\
\text { coefficient } \\
\left(\text { Model B } \mathrm{B}^{\mathrm{e}}\right)\end{array}$ & $\begin{array}{l}\text { Partial } \\
\text { regression } \\
\text { coefficient } \\
(\beta) \pm \mathrm{SE} \\
\left(\text { Model } \mathrm{A}^{\mathrm{d}}\right)\end{array}$ & $\begin{array}{l}\text { Standardized } \\
\text { partial } \\
\text { regression } \\
\text { coefficient } \\
\left(\text { Model } \mathrm{A}^{\mathrm{d}}\right)\end{array}$ & $\begin{array}{l}p \text { value } \\
\left(\text { Model } \mathrm{A}^{\mathrm{d}}\right)\end{array}$ & $\begin{array}{l}\text { Standardized } \\
\text { partial } \\
\text { regression } \\
\text { coefficient } \\
\left(\text { Model } \mathrm{B}^{\mathrm{e}}\right)\end{array}$ \\
\hline \multicolumn{10}{|l|}{ Males } \\
\hline Age & $0.017 \pm 0.051$ & 0. & & 0.746 & -0.036 & $-0.001 \pm 0.014$ & -0.004 & 0.939 & -0.102 \\
\hline FPG & $0.020 \pm 0.006$ & 0.2 & & 0.001 & - & $0.026 \pm 0.021$ & 0.064 & 0.226 & - \\
\hline $\mathrm{HbA1c}$ & $0.654 \pm 0.347$ & 0. & & 0.061 & 0.118 & $0.107 \pm 0.542$ & 0.011 & 0.843 & -0.042 \\
\hline $\mathrm{TC}$ & $0.048 \pm 0.014$ & 0.2 & & 0.001 & -0.040 & $0.010 \pm 0.004$ & 0.124 & 0.020 & 0.101 \\
\hline $\log \mathrm{TG}$ & $1.948 \pm 0.843$ & 0. & & 0.022 & 0.018 & $-0.033 \pm 0.321$ & -0.006 & 0.917 & -0.074 \\
\hline Cre & $6.988 \pm 1.998$ & 0.2 & & $<0.001$ & - & $3.948 \pm 1.243$ & 0.167 & 0.002 & - \\
\hline eGFR & $-0.109 \pm 0.033$ & -0.2 & & 0.001 & $-0.468 * *$ & $-0.039 \pm 0.013$ & -0.160 & 0.004 & $-0.161 *$ \\
\hline $\mathrm{BP}$ & $0.705 \pm 0.678$ & 0. & & 0.299 & -0.052 & $0.420 \pm 0.204$ & 0.116 & 0.040 & 0.046 \\
\hline a-h agent & $2.184 \pm 1.166$ & 0. & & 0.063 & $0.257 *$ & $0.859 \pm 0.428$ & 0.111 & 0.046 & 0.099 \\
\hline BMI & $0.039 \pm 0.059$ & 0. & & 0.513 & 0.014 & $0.116 \pm 0.065$ & 0.097 & 0.073 & 0.106 \\
\hline Smoking $^{\mathrm{a}}$ & $2.136 \pm 1.131$ & 0. & & 0.060 & 0.052 & $0.477 \pm 0.471$ & 0.054 & 0.311 & 0.047 \\
\hline Drinking $^{\mathrm{b}}$ & $0.588 \pm 1.087$ & 0. & & 0.590 & -0.048 & $-0.806 \pm 0.403$ & -0.104 & 0.046 & $-0.157 *$ \\
\hline \multirow[t]{2}{*}{ V event ${ }^{c}$} & $-1.230 \pm 1.347$ & -0. & & 0.363 & $-0.154^{*}$ & $-0.002 \pm 0.577$ & -0.001 & 0.997 & -0.021 \\
\hline & \multicolumn{5}{|l|}{$\mathrm{DM}(n=84)$} & \multicolumn{4}{|c|}{ Non-DM $(n=605)$} \\
\hline \multicolumn{10}{|l|}{ Females } \\
\hline Age & \multicolumn{2}{|c|}{$0.050 \pm 0.025$} & 0.121 & 0.004 & 0.082 & $0.032 \pm 0.008$ & 0.166 & $<0.001$ & 0.086 \\
\hline FPG & \multicolumn{2}{|c|}{$0.002 \pm 0.003$} & 0.031 & 0.609 & - & $-0.006 \pm 0.012$ & -0.020 & 0.626 & - \\
\hline $\mathrm{HbA1c}$ & \multicolumn{2}{|c|}{$0.105 \pm 0.150$} & 0.043 & 0.488 & 0.145 & $-0.133 \pm 0.310$ & -0.020 & 0.668 & -0.054 \\
\hline $\mathrm{TC}$ & \multicolumn{2}{|c|}{$0.003 \pm 0.007$} & 0.026 & 0.665 & 0.032 & $0.004 \pm 0.002$ & 0.077 & 0.060 & 0.076 \\
\hline $\log \mathrm{TG}$ & \multicolumn{2}{|c|}{$0.644 \pm 0.438$} & 0.087 & 0.146 & 0.035 & $0.128 \pm 0.199$ & 0.027 & 0.521 & -0.046 \\
\hline Cre & \multicolumn{2}{|c|}{$5.448 \pm 0.387$} & 0.834 & $<0.001$ & - & $7.057 \pm 0.823$ & 0.342 & $<0.001$ & - \\
\hline eGFR & \multicolumn{2}{|c|}{$-0.105 \pm 0.021$} & -0.523 & $<0.001$ & $-0.400 *$ & $-0.048 \pm 0.007$ & -0.160 & $<0.001$ & $-0.241 * *$ \\
\hline $\mathrm{BP}$ & \multicolumn{2}{|c|}{$0.756 \pm 0.376$} & 0.120 & 0.048 & 0.179 & $0.334 \pm 0.120$ & 0.123 & 0.006 & 0.161 \\
\hline $\mathrm{a}-\mathrm{h}$ agent & \multicolumn{2}{|c|}{$0.377 \pm 0.547$} & 0.043 & 0.492 & 0.030 & $0.235 \pm 0.259$ & 0.039 & 0.365 & -0.041 \\
\hline BMI & \multicolumn{2}{|c|}{$0.095 \pm 0.065$} & 0.157 & 0.153 & 0.064 & $0.050 \pm 0.031$ & 0.066 & 0.110 & 0.065 \\
\hline Smoking $^{\mathrm{a}}$ & \multicolumn{2}{|c|}{$1.096 \pm 0.767$} & 0.085 & 0.157 & 0.155 & $0.469 \pm 0.357$ & 0.053 & 0.190 & 0.073 \\
\hline Drinking $^{\mathrm{b}}$ & \multicolumn{2}{|c|}{$-0.814 \pm 0.605$} & -0.079 & 0.182 & -0.066 & $-0.156 \pm 0.200$ & -0.031 & 0.436 & -0.008 \\
\hline V event ${ }^{c}$ & \multicolumn{2}{|c|}{$1.019 \pm 0.613$} & 0.098 & 0.101 & $0.178 *$ & $0.696 \pm 0.358$ & 0.079 & 0.053 & 0.060 \\
\hline
\end{tabular}

Cre, Serum creatinine; eGFR, estimated glomerular filtration rate by MDRD method; $\mathrm{BP}$, blood pressure $(\mathrm{N}=0$; normal as $\mathrm{SBP}<130 \mathrm{mmHg}$, and $\mathrm{DBP}<80 \mathrm{mmHg}, \mathrm{B}=1$; high level of normal range as $130 \mathrm{mmHg} \leq \mathrm{SBP}<140 \mathrm{mmHg}$, and/or $80 \mathrm{mmHg} \leq \mathrm{DBP}<90 \mathrm{mmHg}$ except for hypertension, $\mathrm{H}=2$; hypertension as $\mathrm{SBP} \geq 140 \mathrm{mmHg}$, or $\mathrm{DBP} \geq 90 \mathrm{mmHg}$ ); a-h agent, anti-hypertensive agent (no $=0$, routine intake $=1)$

a Smoking habits; non-smokers $=0$, current smokers $=1$

${ }^{\mathrm{b}}$ Drinking habits; non-or occasional drinkers $=0$, habitual drinkers $=1$

c Vascular event; history of Myocardial infarction or Cerebral Infarction (not yet $=0$, or have past history $=1$ )

d Model A was adjusted for age and Cre, however, that of eGFR was adjusted for Age

e Model B was adjusted for multi-variables (Age, HbA1c, TC, logTG, eGFR, BP, a-h agent, BMI, Smoking, Drinking and V event)

$* p$ value $<0.05, * * p$ value $<0.001$ 
Table 4 Comparisons of serum total homocysteine level among different subgroups in diabetic subjects according to analysis of covariance

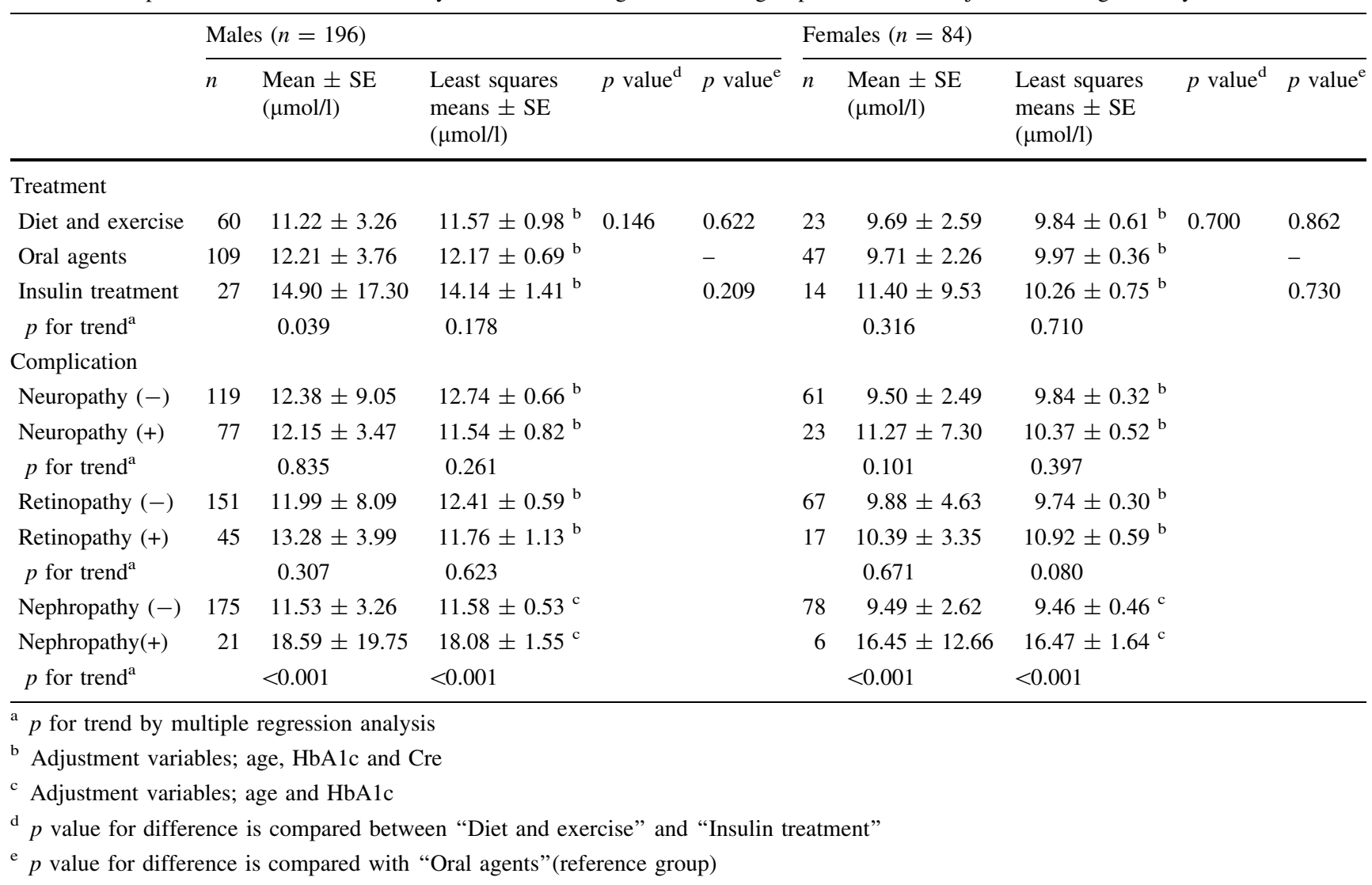

important agents affecting tHcy [33], all the above results were basically unchanged (data were not shown).

\section{Discussion}

First, the association between tHcy and glycemic control, DM treatment type, and diabetic complication were considered. Glycemic status had an independent close association with tHcy in diabetic males only. In this study, the association of tHcy and glycemic control in females was not significant, suggesting that this sex difference was possibly caused by sex hormones. As estrogen inhibits the progression of atherosclerosis [34], shortage of estrogen in males may lead to elevation of tHcy, which promotes thrombogenesis.

Hyperglycemia increases oxidative stress and asymmetric dimethylarginine (ADMA) in the vascular endothelium [35]. Meanwhile, elevated tHcy induces endothelial dysfunction via increased oxidative stress and ADMA [4, 36, 37]. This oxidative stress affecting tHcy may arise as a result of oxidative damage of vascular endothelial cells and increased proliferation of vascular smooth muscle cells after oxidative metabolism of homocysteine to homocysteine and homocysteine thiolactone. However, it remains unclear whether hyperglycemia leads to elevation of tHcy or, conversely whether tHcy leads to hyperglycemia [2]. For males in this study, the glycemic status had close association with tHcy in DM but not in non-DM. Therefore, tHcy may increase after the onset of DM.

Although we did not measure plasma insulin levels in this study, some previous studies have shown the positive association between insulin-resistant status and tHcy, and indicated that insulin levels may affect the activity of enzymes involved in homocysteine metabolism [38]. Fonseca et al. [38] have reported that insulin treatment in rats caused a reduction of the enzyme cystathionine $\beta$-synthase and increasing MTHFR activity, leading to elevated tHcy. This may suggest that not only chronic hyperinsulynemia but also acute hyperinsulynemia by insulin injection increase tHcy. In this study, DM treatment levels after adjustment for HbA1c, age, and Cre were not significantly associated with tHcy. However, tHcy of the subjects receiving insulin therapy was relatively high compared with the other two categories of treatment. These findings were consistent with previous studies. 
With respect to the effects of diabetic complications, a strong association between nephropathy and tHcy was found in this study. This finding was consistent with the results from previous studies [39-41]). It has been revealed that the deterioration of renal function is associated with elevated tHcy. The transformation of methionine to homocysteine occurs via a demethylation pathway, providing a methyl group with glycocyamine in the creation of creatinine [42]. Thus, in renal metabolism, homocysteine is closely linked with formation of creatinine [43], through decreased renal homocysteine excretion, impaired renal metabolism, or generally reduced B vitamins status in renal failure [44]. Therefore, Elias and Eng [24] suggested that adequate management of tHcy could enable prevention of renal dysfunction.

Second, it is noteworthy that eGFR and Cre within their normal ranges were strongly associated with tHcy in every group, DM and non-DM, in both sexes of this study. These results were consistent with a report by Abdella, et al. [45]. Subtle changes in eGFR and Cre after adjustment reflect minimal abnormality of renal function, even though within their normal ranges they may affect tHcy. It has been established that the incidence and mortality of CKD increase with the deterioration of eGFR [46]. This study suggests that the strong association between tHcy and eGFR may represent a link between heart and kidney. There is a possibility that a slight change of tHcy in DM patients or prediabetics can indicate early microangiopathy of the kidney. However a previous study gave conflicting results, showing that tHcy in early diabetic nephropathy was not increased [47]. As another possibility, elevated tHcy might cause microvascular endothelial damage in a renal circulation. Clarification of these mechanisms may be an important issue in future research.

One of the limitations of this study is that we did not have information on nutrition status of vitamin B6, B12 and folic acid, or gene type such as MTHFR. Furthermore, since this study was based on a cross-sectional design, many statistical associations with tHcy found in this study could not be correctly interpreted.

In the future, further follow-up or interventional studies will be necessary in order to increasingly clarify the significance of tHcy in diabetics.

In conclusion, these findings suggest there are some differences in the factors associated with tHcy between diabetics and non-diabetics, and between males and females. Glycemic status in DM was more strongly associated with tHcy in males than in females. There is, therefore, circumstantial evidence a prevention and treatment regimen may be required for elevated tHcy. Because tHcy was strongly associated with eGFR and Cre even within the normal ranges, tHcy may have important implications regarding the microangiopathy of the kidney and atherosclerosis.

\section{References}

1. Stipanuk MH. Sulfur amino acid metabolism: pathways for production and removal of homocysteine and cysteine. Annu Rev Nutr. 2004;24:539-77.

2. Welch GN, Loscalzo J. Homocysteine and atherothrombosis. N Engl J Med. 1998;338:1042-50.

3. Maxwell SR. Coronary artery disease-free radical damage, antioxidant protection and the role of homocysteine. Basic Res Cardiol. 2000;95(suppl1):165-71.

4. Weiss N. Mechanisms of increased vascular oxidant stress in hyper homocysteinemia and its impact on endothelial function. Curr Drug Metab. 2005;6:27-36.

5. The Homocysteine Studies Collaboration. Homocysteine, risk of ischemic heart disease, stroke. JAMA. 2002;288:2015-22.

6. Eikelboom JW, Lonn E, Genest J Jr, Hankey G, Yusuf S. Homocyst(e)ine and cardiovascular disease: a critical review of the epidemiologic evidence. Ann Intern Med. 1999;131:363-75.

7. Soinio M, Marniemi J, Laakso M, Rönnemaa T. Elevated plasma homocysteine level is an independent predictor of coronary heart disease events in patients with type 2 diabetes mellitus. Ann Intern Med. 2004;140:94-100.

8. Iso H, Moriyama Y, Sato S, Kitamura A, Tanigawa T, Shimamoto T, et al. Serum total homocysteine concentrations and risk of stroke and its subtypes in Japanese. Circulation. 2004; 109:2766-72.

9. Kawamoto R, Kajiwara T, Oka Y, Takagi Y. An association between plasma homocysteine concentrations and ischemic stroke in elderly Japanese. J Atheroscler Thromb. 2001;9:121-5.

10. Schneede J, Refsum H, Ueland PM. Biological and environmental determinants of plasma homocysteine. Semin Thromb Hemost. 2002;26:263-79.

11. Refsum H, Smith AD, Ueland PM. Facts and recommendations about total homocysteine determinations: an expert opinion. Clin Chem. 2004;50:3-32.

12. Stanger O, Herrmann W, Pietrzik K, Fowler B, Geisel J, Wegel $\mathrm{M}$, et al. DACH-LIGA homocysteine e.V.: DACH-LIGA homocysteine (german, austrian and swiss homocysteine society): consensus paper on the rational clinical use of homocysteine, folic acid and B-vitamins in cardiovascular and thrombotic disease: guidelines and recommendations. Clin Chem Lab Med. 2004;42:113-6.

13. Jacques PF. Determinants of plasma total homocysteine concentration in the Framingham offspring cohort. Am J Clin Nutr. 2001;73:613-21.

14. Diakoumopoulou E, Tentolouris N, Kirlaki E, Perrea D, Kitsou E, Katsilambros N, et al. Plasma homocysteine levels in patients with type 2 diabetes in a Mediterranean population: relation with nutritional and other factors. Nutr Metab Cardiovasc Dis. 2005;15:109-17.

15. Kluijtmans LA, Kastelein JJ, Lindemans J, Boers GH, Heil SG, Blom HJ, et al. Thermolabile methylenetetrahydrofolate reductase in coronary artery disease. Circulation. 1997;96:2573-7.

16. Nygård O, Vollset SE, Refsum H, Stensvold I, Tverdal A, Kvåle $\mathrm{G}$, et al. Total plasma homocysteine and cardiovascular risk profile. The Hordaland homocysteine study. JAMA. 1995; 274:1526-33.

17. Hurst RT, Lee RW. Increased incidence of coronary atherosclerosis in type 2 diabetes mellitus: mechanisms and management. Ann Intern Med. 2003;139:824-34.

18. Fox CS, Coady S, Sorlie PD, Levy D, Meigs JB, Savage PJ, et al. Trends in cardiovascular complications of diabetes. JAMA. 2004;292:2495-9.

19. Sone H, Katagiri A, Ishibashi S, Abe R, Saito Y, Yamada N, et al. JD Study Group. Effects of lifestyle modifications on patients 
with type 2 diabetes: the Japan diabetes complications study (JDCS) study design, baseline analysis and three year-interim report. Horm Metab Res. 2002;34:509-15.

20. Fujishima M, Kiyohara $\mathrm{Y}$, Kato I, Ohmura T, Iwamoto $H$, Yoshitake $\mathrm{T}$, et al. Diabetes and cardiovascular disease in a prospective population survey in Japan: the Hisayama study. Diabetes. 1996;45(supp13):S14-6.

21. Emoto M, Kanda H, Shoji T, Kawagishi T, Komatsu M, Nishizawa $\mathrm{Y}$, et al. Impact of insulin resistance and nephropathy on homocysteine in type 2 diabetes. Diabetes Care. 2001;24:5338.

22. Mazza A, Bossone E, Mazza F, Distante A. Reduced serum homocysteine levels in type 2 diabetes. Nutr Metab Cardiovasc Dis. 2005;15:118-24.

23. Schalinske KL. Interrelationship between diabetes and homocysteine metabolism: hormonal regulation of cystathionine betasynthase. Nutr Rev. 2003;61:136-8.

24. Elias AN, Eng S. Homocysteine concentrations in patients with diabetes mellitus-relationship to microvascular and macrovascular disease. Diabetes Obes Metab. 2005;7:117-21.

25. Masaki T, Anan F, Anai M, Higuchi K, Tsubone T, Yoshimatsu $\mathrm{H}$, et al. Hyperhomocysteinemia is associated with visceral adiposity in Japanese patients with type 2 diabetes mellitus. Diabetes Res Clin Pract. 2007;77:168-73.

26. Imai E, Horio M, Nitta K, Yamagata K, Iseki K, Matsuo S, et al. Estimation of glomerular filtration rate by the MDRD study equation modified for Japanese patients with chronic Kidney disease. Clin Exp Nephrol. 2007;11:41-50.

27. The expert committee on the diagnosis and classification of diabetes mellitus. Report of the expert committee on the diagnosis and classification of diabetes mellitus. Diabetes Care. 1997;20:1183-97.

28. Hatakeyama I, Maruko T, Ida Y, Tsuruoka A, Maehata E. Fundamental and clinical evaluation for glycosylated hemoglobin measurement kit "Determiner HbA1c" using latex agglutination immunoassay. Med Pharm. 1999;41:1181-6.

29. Sakurai T. Fundamental study on measurement of total cholesterol by ultra violet-end (UV-End) method using cholesterol dehydrogenase (CDH). Jpn J Med Techol. 1998;47:747-52.

30. Shibuya Y, Okabe H. Triglyceride. Nippon Rinsho. 1995;53(suppl):606-10.

31. Chobanian and the National High Blood Pressure Education Program Coordinating Committee, et al. Seventh report of the joint national committee on prevention, detection, evaluation, and treatment of high blood pressure. Hypertension. 2003;42:120652.

32. The committee of Japan Diabetes Society for the Diagnostic Criteria of Diabetes Mellitus, Kuzuya T, Kadowaki T, et al. Report of the committee of japan diabetes society on the classification and diagnostic criteria of diabetes mellitus. J Japan Diab Soc. 1999;42:385-404.

33. Hoogeveen EK, Kostense PJ, Jakobs C, Bouter LM, Heine RJ, Stehouwer CD. Does metformin increase the serum total homocysteine level in non-insulin-dependent diabetes mellitus? J Intern Med. 1997;242:389-94.
34. Wouters MG, Moorrees MT, van der Mooren MJ, Blom HJ, Boers GH, Eskes TK, et al. Plasma homocysteine and menopausal status. Eur J Clin Invest. 1995;25:801-5.

35. Xiong $\mathrm{Y}, \mathrm{Fu} \mathrm{Y}, \mathrm{Fu} \mathrm{S}$, Zhou H. Elevated levels of the serum endogenous inhibitor of nitric oxide synthase and metabolic control in rats with streptozotocin-induced diabetes. J Cardiovasc Pharmacol. 2003;42:191-6.

36. Antoniades C, Tousoulis D, Marinou K, Vasiliadou C, Tentolouris C, Stefanadis C, et al. Asymmetrical dimethylarginine regulates endothelial function in methionine-induced but not in chronic homocysteinemia in humans: effect of oxidative stress and proinflammatory cytokines. Am J Clin Nutr. 2006;84:781-8.

37. Björck J, Hellgren M, Råstam L, Lindblad U. Associations between serum insulin and homocysteine in a Swedish populationa potential link between the metabolic syndrome and hyperhomocysteinemia: the Skaraborg project. Metabolism. 2006;55:1007-13.

38. Fonseca V, Dicker-Brown A, Ranganathan S, Song W, Barnard RJ, Kern PA, et al. Effects of a high-fat-sucrose diet on enzymes in homocysteine metabolism in the rat. Metabolism. 2000;49:736-41.

39. Abdella N, Mojiminiyi OA, Akanji AO. Homocysteine and endogenous markers of renal function in type 2 diabetic patients without coronary heart disease. Diabetes Res Clin Pract. 2000; 50:177-85.

40. Stabler SP, Estacio R, Jeffers BW, Cohen JA, Allen RH, Schrier $\mathrm{RW}$. Total homocysteine is associated with nephropathy in noninsulin-dependent diabetes mellitus. Metabolism. 1999;48:1096101.

41. Ozmen B, Ozmen D, Turgan N, Habif S, Mutaf I, Bayindir O. Association between homocysteine and renal function in patients with type 2 diabetes mellitus. Ann Clin Lab Sci. 2002;32:279-86.

42. Mudd SH, Poole JR. Labile methyl balances for normal humans on various dietary regimens. Metabolism. 1975;24:721-35.

43. Davies L, Wilmshurst EG, McElduff A, Gunton J, Clifton-Bligh $\mathrm{P}$, Fulcher GR. The relationship among homocysteine, creatinine clearance, and albuminuria in patients with type 2 diabetes. Diabetes Care. 2001;24:1805-9.

44. van Guldener C, Stehouwer CD. Homocysteine metabolism in renal disease. Clin Chem Lab Med. 2003;41:1412-7.

45. Abdella NA, Mojiminiyi OA, Akanji AO, Moussa MA. Association of plasma homocysteine concentration in subjects with type 2 diabetes mellitus. Acta Diabetol. 2002;39:183-90.

46. Nakamura K, Okamura T, Hayakawa T, Kadowaki T, Kita Y, Ueshima H, et al. The NIPPON DATA90 Research Group. Chronic kidney disease is a risk factor for cardiovascular death in a community-based population in Japan: NIPPON DATA90. Circ J. 2006;70:954-9.

47. Agardh CD, Agardh E, Andersson A, Hultberg B. Lack of association between plasma homocysteine levels and microangiopathy in type 1 diabetes mellitus. Scand J Clin Lab Invest. 1994;54:637-41. 